\title{
Serial ECG Analysis after Myocardial Infarction: When Heart Failure Develops, the ECG Becomes Increasingly Discordant
}

\author{
Marjolein C de Jongh, Arie C Maan, Enno T van der Velde, Cees A Swenne \\ Leiden University Medical Center, Leiden, The Netherlands
}

\begin{abstract}
This study sought to identify an ECG variable that can be used to detect emerging heart failure (HF) in patients after myocardial infarction (MI). We adopted the spatial QRS-T angle (SA) as a measure of ECG concordance, and hypothesized that the ECG of patients who develop HF becomes less concordant. We computed SA in ECGs of 20 patients (15/5 male/female, mean $\pm S D$ age $64 \pm 10 \mathrm{yrs})$, at baseline (6 months after MI) and 3.8 2.4 yrs later, when they presented with $\mathrm{HF}$. SA increased from $97 \pm 36^{\circ}$ to $119 \pm 34^{\circ}(P=0.003)$. SA decreased only in 4 pts. We conclude that SA is potentially a suitable ECG monitoring variable for early detection of HF after MI.
\end{abstract}

\section{Introduction}

Heart failure (HF) is a major public health issue. In 1997, HF was declared an emerging epidemic[1]. In a 2013 publication[2], the worldwide prevalence of HF was estimated 23 million; a review published in 2016 gives an estimated worldwide prevalence of 38 million[3]. Major etiologies underlying HF are myocardial infarction (MI), diabetes, and high blood pressure[4]; more than $50 \%$ of patients presenting with HF have a history of MI[5].

According to the ACC/AHA heart failure staging system[6], there are four stages of HF:

- $\quad$ Stage A: Patient at high risk for developing HF with no structural disorder of the heart.

- $\quad$ Stage B: Patient with structural disorder of the heart without symptoms of HF.

- $\quad$ Stage C: Patient with past or current symptoms of HF associated with underlying structural heart disease.

- Stage D: Patient with end-stage disease who requires specialized treatment strategies.

In the setting of prevention of HF, the transient between stage $B$ and stage $C$ is of crucial importance. Usually, patients, after having experienced a myocardial infarction, and, hence, from then on being in stage $B$, are treated with beta-blockers, ACE inhibitors, statins, anti-thrombotics and lifestyle intervention. Typical additional HF medication like diuretics and aldosterone receptor blockers, and fluid management are only introduced when clinical signs of HF have become immanent[7] and the patient is already in HF stage C. Obviously, waiting for symptoms of HF implies that the process of developing $\mathrm{HF}$ has already been started and that potentially structural damage (ventricular remodelling) has already occurred. Our current research is directed towards the ECG changes during the transient period from HF stage B to stage C. It is likely that any structural and electrically functional change of the heart is associated with a change in the ECG. If, at the onset of HF stage C, the ECG has changed with respect to the $\mathrm{HF}$ stage $\mathrm{B}$, we have a potential means of monitoring the patient during HF stage $\mathrm{B}$ in order to detect a possible threatening transition to HF stage C.

In the normal heart, depolarisation and repolarisation have a fixed order, dictated by the conduction system and the action potential duration distribution. Left-ventricular endocardial myocytes are activated first and epicardial myocytes are activated last. However, endocardial action potential durations are longer then epicardial action potential durations. Hence, repolarisation starts at the epicardium and follows the opposite direction as depolarisation. As a consequence, the ECG amplitudes during repolarisation have, in many ECG leads, the same sign as the ECG amplitudes during depolarisation, and, in 3D space, the QRS- and T-axes assume about the same direction and constitute an acute angle. This characteristically healthy ECG is called concordant. The more the intricate physiology of the electrical activity of the heart is disturbed, e.g., by corruption of the conduction by fibrosis and hypertrophy (both prominent structural changes associated with HF), the larger the spatial angle (SA) between the QRS- and the T-axes and the more discordant the ECG. In other words, the SA, as a measure for concordance/discordance, and assumed to increase with developing HF, could potentially be an ECG variable suited for serial ECG analysis with the purpose to monitor patients for early HF detection.

In our current study, we focused on patients who experienced a myocardial infarction, became initially stable and had no symptoms of HF, and who developed later overt symptoms of HF for which they were treated. We hypothesized that the ECG in the HF stage was less concordant / more discordant then the ECG post-MI. 


\section{Methods}

In our electronic patient database, we searched for patients who initially presented with an acute myocardial infarction, became stable after recovery, and presented later with symptoms of $\mathrm{HF}$. After acute myocardial infarction, patients in our hospital enter routinely the "Mission Infarction" protocol[8]. This protocol includes follow-up visits 1, 3, 6 and 12 months after the acute event, including echocardiography and bicycle ergometry. All patients who were stable after 6 months were candidates for our study; selection criteria were:

- $\quad$ ejection fraction $>35 \%$

- no decompensation and/or fluid retention

- $\quad$ no decreased exercise tolerance.

Diuretics were allowed in patients who had such medication already before their myocardial infarction occurred, e.g., because of hypertension, however, newly prescribed diuretics in the post-infarction period were considered as a sign of potential HF, and such patients were not included. The standard 12-lead ECG that was made at the 6-months visit was taken as reference ECG that characterized the electrical heart function of the patient after myocardial infarction (ECG-MI).

Candidate patients for our study, with stabilized myocardial infarction 6 months after the acute event, were included in our study when they later presented with clinical signs of HF (fluid retention or decreased exercise tolerance) without being decompensated. The standard 12lead ECG that was made at the initial visit to our HF outpatient clinic was taken as follow-up ECG that characterized the electrical heart function of the patient at initial presentation with HF (ECG-HF).

Patients with a technically deficient ECGs, non-sinus rhythm, arrhythmias or pacemaker rhythm were excluded. Otherwise abnormal ECGs were tolerated.

All ECGs were analyzed by our research ECG analysis program LEADS[9]. The 12-lead ECG was converted to a vectorcardiogram (VCG) by using the Kors matrix[10]. The spatial QRS-T angle (SA) was computed in the VCG. SA values measured in ECG-MI and in ECG-HF were compared by a single-sided paired t test and by correlation analysis at the $5 \%$ significance level.

\section{Results}

We studied 20 patients (15/5 male/female), see Table 1 for patient characteristics.

Table 1. Patient characteristics.

\begin{tabular}{lll}
\hline $\mathrm{N}=20$ (15/5 male/female) & Mean \pm SD & Range \\
\hline $\begin{array}{l}\text { Age at inclusion (y) } \\
\begin{array}{l}\text { Time between ECG-MI } \\
\text { and ECG-HF (y) }\end{array}\end{array}$ & $64 \pm 10$ & $38-79$ \\
\hline
\end{tabular}

Figure 1 is an example of a baseline ECG after myocardial infarction, ECG-MI, and an ECG made 50 months later, when the patient presented for the first time at the HF outpatient clinic, ECG-HF. This male patient had an inferoposterolateral infarction at the age of 42 years and became stable with an ejection fraction of $42 \%$. The SA at baseline was $30^{\circ}$, SA had increased till $101^{\circ}$ when the patient presented with HF. In this patient, most changes occurred in the $\mathrm{T}$ wave, but other patients showed also QRS changes.

In the complete study group, SA increased on average by $22^{\circ}$, see Table 2 . In only $4 / 20$ patients, SA decreased. Figure 2 shows a scatterplot of all data.

Table 2. SA at baseline and SA at presentation with HF; the difference is statistically significant $(P=0.003)$.

\begin{tabular}{lll}
\hline $\mathrm{N}=20$ & Mean \pm SD & Range \\
\hline SA in ECG-MI $\left(^{\circ}\right)$ & $97 \pm 36$ & $30-160$ \\
SA in ECG-HF $\left(^{\circ}\right)$ & $119 \pm 34$ & $43-166$ \\
\hline
\end{tabular}

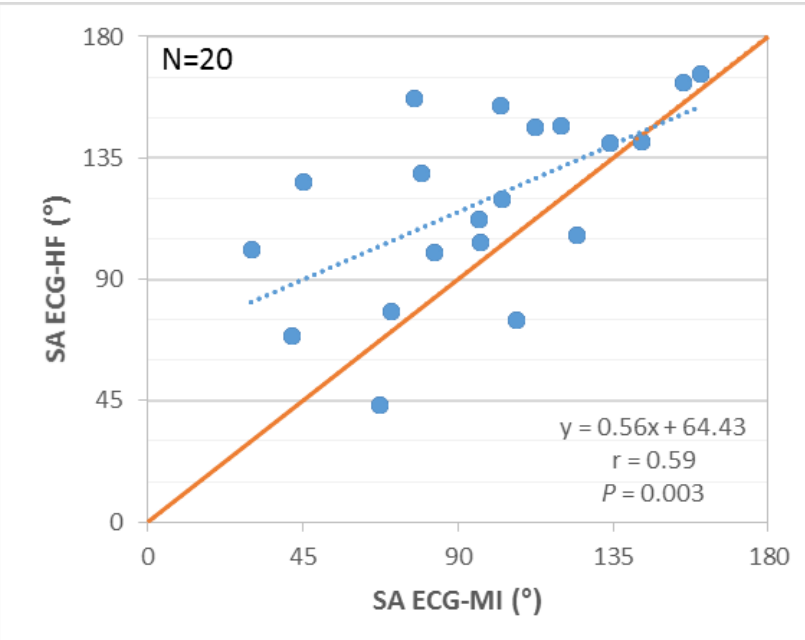

Figure 2. SA at baseline (SA ECG-MI) and after HF had developed (SA ECG-HF), with a linear regression line (blue, dotted), and with the line of identity (red).

\section{Discussion}

We investigated the difference in concordance between ECGs made in patients who were stable 6 months after MI and who presented later for the first time at the outpatient clinic with HF symptoms. As a measure of concordance we adopted the spatial angle between the QRS- and T axes, SA. The larger SA, the less concordant / more discordant the ECG. We found in 20 patients after an average period of 3.8 years between ECG-MI and ECG-HF, a significant increase of SA by $22^{\circ}$ which may be interpreted as a decreased concordance / increased discordance of the 


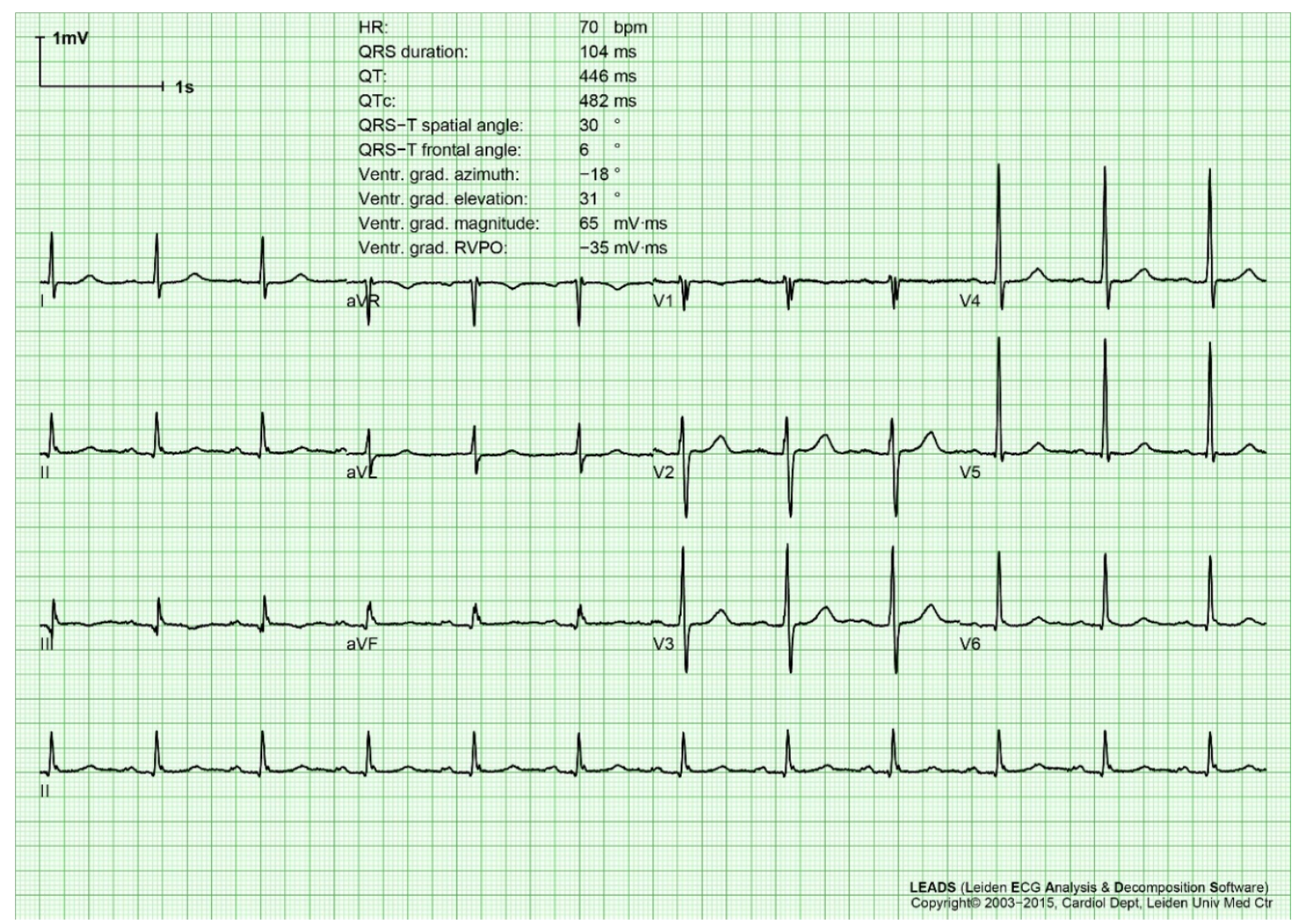

Figure 1, panel A. ECG-MI, made 6 months after myocardial infarction in subject \#13.

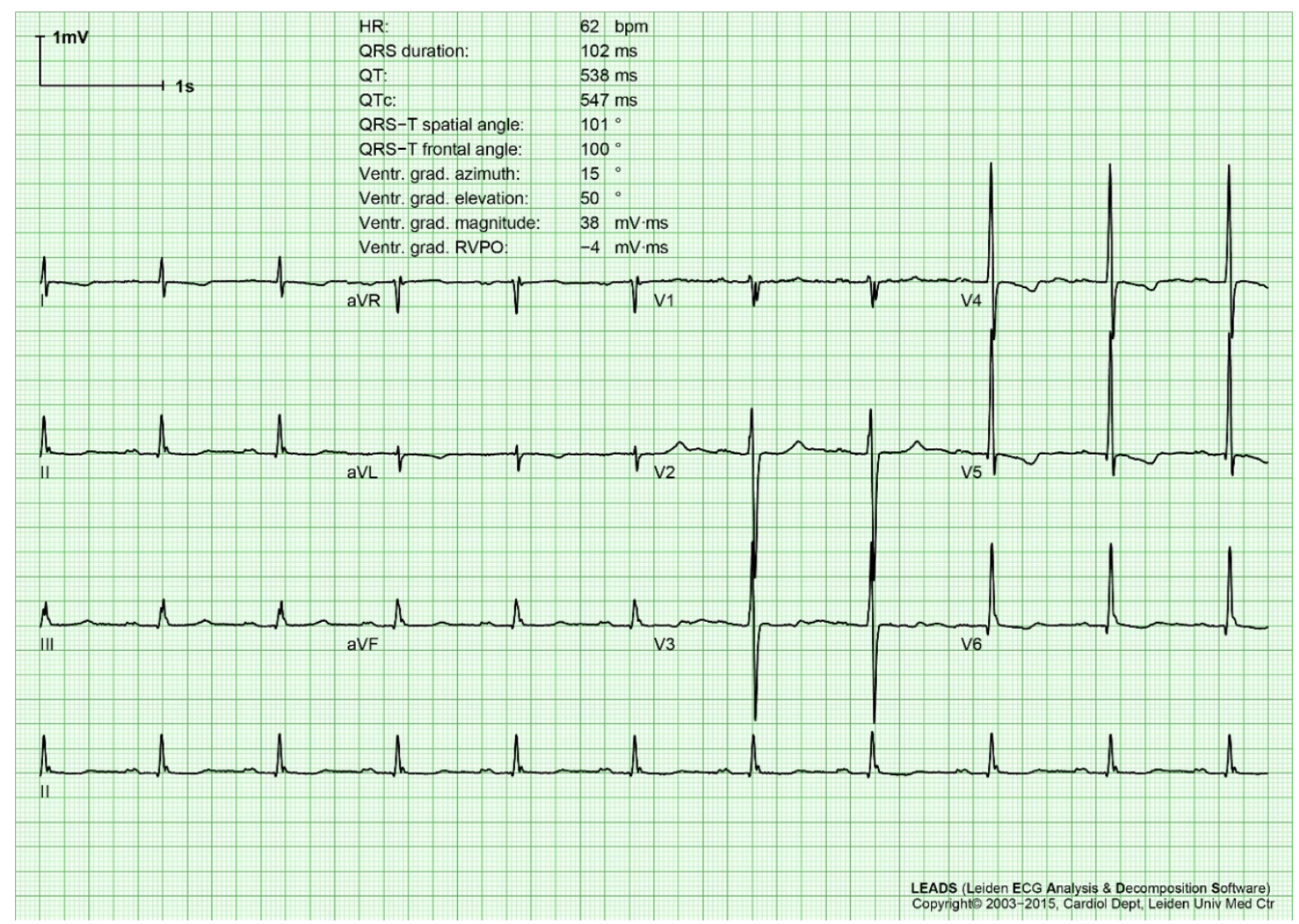

Figure 1, panel B. ECG-HF, made in subject \#13 at first presentation with HF, 50 months after ECG-MI had been made. 
ECGs of these patients in the beginning stage of HF compared to their earlier stabilized situation after MI.

The VCG is a 3D representation of the electrical activity of the heart [11]. In this representation, the QRS- and Taxes both assume a direction in 3D space, and the so-called spatial angle, SA, is actually the planar angle between these axes. In normal hearts, the QRS and $\mathrm{T}$ axes form an acute angle in concordant situations and an obtuse angle in discordant situations. This must be seen as a continuum, and there is even considerable overlap between SA in normal ECGs and SA in abnormal ECGs. Actually, the upper limit of SA in normal subjects is around $100^{\circ}[12]$. However, there are good reasons to suppose that SA for a given subject is fairly constant in time, and, as a consequence, an intra-individual change in SA implies a change in the cardiac electrical activity that makes the individual suspect of having an active pathophysiologic process. After MI, patients have a strongly increased risk of developing HF. HF is known to be accompanied by cardiac enlargement, hypertrophy and fibrosis; all these are factors that corrupt conduction and increase action potential durations in affected areas, thus having a detrimental influence on the normal cardiac electrical activity, logically leading to decreased concordance / increased discordance. Hence, an increase of SA makes the patient suspect of developing HF.

Patients after myocardial infarction may sometimes have a normal ECG, but often the ECG is abnormal due to the damage caused by the infarction. Figure 1 shows that several patients had an ECG-MI with rather large SA values. This means that their ECG-MI is already discordant in a certain sense. However, it appears that in all but 4 patients SA increases further when HF has developed. Even those in whom SA decreased, the amount of decrease is limited. Hence, the phenomenon that we observed was pervasively present in the study group. This renders SA a promising monitoring variable for patients who are suspect of developing $\mathrm{HF}$.

We have not been able to study the intra-individual trends of SA in the time period in between ECG-MI and ECG-HF. In this period, most patients have check-ups with their general practitioner, do not visit a cardiology outpatient clinic, and ECGs, if any, are only infrequently made and are not in our hospital database. Hence, we do not know if the ECG changes in our study group occurred suddenly, e.g., within weeks, or gradually, e.g., over months or years. Given the average time interval (3.8 years) after which patients presented with HF we can imagine that a yearly follow-up ECG could be very useful. Upon detection of an SA increase, an extra echocardiogram could be made to check for possible developing HF. Further research is needed to corroborate such a preventive protocol for early detection of emerging HF; such research would also yield a control group of patients who do not develop HF after MI.

\section{References}

[1] Braunwald E. Shattuck lecture--cardiovascular medicine at the turn of the millennium: triumphs, concerns, and opportunities. The New England Journal of Medicine. 1997;337:1360-9.

[2] Roger VL. Epidemiology of heart failure. Circulation Research. 2013;113:646-59.

[3] Ziaeian B, Fonarow GC. Epidemiology and aetiology of heart failure. Nature Reviews Cardiology. 2016;13:368-78.

[4] Kemp CD, Conte JV. The pathophysiology of heart failure. Cardiovascular Pathology : The Official Journal of the Society for Cardiovascular Pathology. 2012;21:365-71.

[5] Mosterd A, Hoes AW. Clinical epidemiology of heart failure. Heart. 2007;93:1137-46.

[6] Hunt SA, Baker DW, Chin MH, Cinquegrani MP, Feldman AM, Francis GS, et al. ACC/AHA Guidelines for the evaluation and management of chronic heart failure in the adult: Executive summary. Circulation. 2001;104:29963007.

[7] Ponikowski P, Voors AA, Anker SD, Bueno H, Cleland JG, Coats AJ, et al. 2016 ESC Guidelines for the diagnosis and treatment of acute and chronic heart failure: The Task Force for the diagnosis and treatment of acute and chronic heart failure of the European Society of Cardiology (ESC). European Heart Journal. 2016;37:2129-200.

[8] Liem SS, van der Hoeven BL, Oemrawsingh PV, Bax JJ, van der Bom JG, Bosch J, et al. MISSION!: Optimization of acute and chronic care for patients with acute myocardial infarction. American Heart Journal. 2007;153:14 e1-1.

[9] Draisma HHM, Swenne CA, van de Vooren H, Maan AC, Hooft van Huysduynen B, van der Wall EE, et al. LEADS: An interactive research oriented ECG/VCG analysis system. Computers in Cardiology. 2005;32:515-8.

[10] Kors JA, van Herpen G, Sittig AC, van Bemmel JH. Reconstruction of the Frank vectorcardiogram from standard electrocardiographic leads: diagnostic comparison of different methods. European Heart Journal. 1990;11:1083-92.

[11] Man S, Maan AC, Schalij MJ, Swenne CA. Vectorcardiographic diagnostic \& prognostic information derived from the 12-lead electrocardiogram: Historical review and clinical perspective. Journal of Electrocardiology. 2015;48:463-75.

[12] Scherptong RW, Henkens IR, Man SC, Le Cessie S, Vliegen HW, Draisma HH, et al. Normal limits of the spatial QRS-T angle and ventricular gradient in 12-lead electrocardiograms of young adults: dependence on sex and heart rate. Journal of Electrocardiology. 2008;41:648-55.

Address for correspondence.

Cees A. Swenne, $\mathrm{PhD}$

Cardiology Department

Leiden University Medical Center

PO Box 9600, 2300 RC Leiden, The Netherlands

c.a.swenne@lumc.nl 